\title{
Atypical onset of refractive accommodative esotropia: A case report
}

\author{
Feray Koc ${ }^{1}$, Figen Tokucoglu ${ }^{2}$ \\ ${ }^{1}$ Neuro-ophthalmology department of Izmir Ataturk Education and Research Hospital, Izmir, Turkey \\ ${ }^{2}$ Neurology department of Izmir Tepecik Education and Research Hospital, Izmir, Turkey \\ Email address: \\ dr_feray@yahoo.com (F. Koc), fesmeli@hotmail.com (F. Tokucoglu)
}

\section{To cite this article:}

Feray Koc, Figen Tokucoglu. Atypical Onset of Refractive Accommodative Esotropia: A Case Report. Clinical Medicine Research. Vol. 3, No. 6, 2014, pp. 203-205. doi: 10.11648/j.cmr.20140306.18

\begin{abstract}
A 27 year old female presented with symptoms of diplopia, headache, ptosis, ataxia, and macropsia. She was subjected to extensive and repetitive medical evaluations on 3 separate occasions over a 2 year period resulting in a delay in diagnosis, expense of the evaluations and unnecessary treatment. Ophthalmologic consultation uncovered a delayed onset of refractive accommodative esotropia. Signs and symptoms of refractive accommodative esotropia can simulate other neurologic diseases in adults.
\end{abstract}

Keywords: Accommodation, Diplopia, Adult Strabismus, Hypermetropia

\section{Introduction}

Refractive accommodative esotropia typically presents during early childhood, having its usual onset between 18-48 months of age [1-2]. The onset of refractive accommodative esotropia in the second or third decade is uncommon, if it is not precipitated by a brief period of occlusion [3-6].Children usually present for treatment of a sudden onset of esotropia. Complaints of symptoms of refractive accommodative esotropia in young children are uncommon and for this reason ophthalmologists are not familiar to these complaints. Herein we present a young adult who presented with complaints of diplopia, headache, ptosis, ataxia, and macropsia. Any acute esotropia with the complaint of diplopia of sudden onset, may require a neurologic evaluation. In adults, oculomotor nerve paresis, extraocular muscle diseases, neuromuscular junction diseases, acute acquired strabismus types and rarely intra cranial mass lesions or malformations might cause acute esotropia [4,5,711]. The patient was evaluated on several occasions over a 2 year span for neurologic conditions and was ultimately found to have refractive accommodative esotropia. The atypical age of onset, and unfamiliarity of the initial examining ophthalmologist with the possibility of the patient having a delayed onset of refractive accommodative esotropia as a cause for her symptoms prompted referral to the neurology service.
The purpose of this report is to make physicians aware that patients with refractive accommodative esotropia can mimic many neurologic conditions and that refractive accommodative esotropia should be considered when evaluating young adults with an onset of diplopia and headache.

\section{Case History}

The principles in the declaration of Helsinki was followed for this report and the patient consent was taken.

A 27 year old woman was seen in consultation at the request of the neurology service at our institution. Her chief complaint was diplopia. It was binocular, horizontal, occurring in all gaze positions for the past two years, intermittent at first but becomes almost continuous now. Her associated signs and symptoms were headache, unilateral macropsia, ataxia, unilateral or bilateral variable ptosis and abduction deficit. These complaints were also occuring intermittently. The patient was initially evaluated by an ophthalmologist who considered the cause for the symptoms as neurologic, and referred her for neurologic evaluation She was hospitalized for the third time for evaluation of these complaints.

In her past medical history she described fainting few times at 15 years old with unknown etiology. She had no other complaint in the review of systems. She did not give any disease history in her family. There was no diabetes 
mellitus or coronary artery disease history in the family. Socially she was single and working as an accountant.

The past medical history of present ilness, abstracted from the previous neurology clinical records recorded "variable ocular motility findings", "an esotropia with lateral rectus restriction", variable blepharoptosis, unilateral on some evaluations and bilateral on others and normal fundi.

Multiple laboratory studies including hematology, urine, blood chemistry, thyroid function testing, chest roentgenogram, and evaluation of the cerebral spinal fluid were normal. Since the fasting blood glucose levels were within normal levels in several occasions, HbA1c levels were not ordered. Urine and blood tests showing kidney functions were also within normal limits. Immune markers for vasculitis and serologic testing for HIV, syphilis, herpes, and brucellosis were also negative. CT scan of the brain, cervical MRI, vertebral MRI, cavernous sinus MRI scans and MRA were also normal. The brain MRI showed few 2 millimeter T2 hyper intense lesions in right frontotemporal region which were not considered to be associated with this patients symptoms. A slight increase in latency of the visual evoked potential (VEP) was detected. Other neurologic nerve transmission tests were normal. Neostigmine test, single fiber EMG test and Ach R-(receptor) antibody titers were negative for ocular myasthenia gravis.

Following this evaluation, it was suspected that she had early phases of a demyelinating process and was treated with pulsed corticosteroid therapy for five days. She showed some improvement from the corticosteroid treatment.

Symptoms reoccurred 6 months later. She was hospitalized again and reevaluated. Similar findings and treatment were given.

On the third admission, an ophthalmology consultation was requested. Evaluation showed, an uncorrected visual acuity of $6 / 7.5 \mathrm{OD}$ and $6 / 6 \mathrm{OS}$. A non-cycloplegic refraction measured with an auto refractor was OD: - 1.50, and OS: - 1.00 OD. Manifest refraction, using trial lenses, showed variable responses from the patient. The pupils were equal. Responses to light and accommodation were normal. There was no relative afferent pupillary defect On the motility evaluation, there was an intermittent small angle esotropia which increased in lateral gaze but decreased in measurement at distance fixation as compared to the near measurement. The angle of esodeviation measured in the primary gaze position was variable and ranged from 2-6 prism diopters (PD) at distance and 12-16 PD at near. Abduction showed -2 restriction in abduction of each eye. The near point of accommodation was $20 \mathrm{~cm}$.

There was mild bilateral blepharoptosis with normal levator function and no evidence of fatigue. The ophthalmoscopic evaluation of the fundi was normal.

A refraction under cycloplegia revealed +4.00 diopters of hypermetropia in both eyes. The patient was prescribed +3.00 diopter lenses and was encouraged to wear them full time. Visual acuity with correction was $6 / 6$ in each eye

The patient was evaluated one month later. She was wearing her glasses full time and was free from symptoms; the esotropia had resolved. At this visit, her mother accompanied her and was observed to be wearing glasses to correct a high hypermetropic refractive error. On questioning, the patient's mother related that her other daughter had developed esotropia and wore glasses when she was a child.

This patient has now been followed for two and a half years. She remains complaint free.

\section{Discussion}

Sudden onset of horizontal diplopia in an adult is always a concern and warrants a thorough evaluation by a neurologist. Cranial nerve paresis or palsy, intracranial mass, hydrocephalus, myasthenia gravis, orbital pathology and decompensated strabismus should be considered in the evaluation [4,5,7-11]. Though our case had an ophthalmologic problem, her unusually late presentation, missing history about the strabismus in the family and unusual symptoms misled her general ophthalmologist and caused her referral to the neurology clinic. The onset of refractive accommodative esotropia in the second or third decade is uncommon, if it is not precipitated by a brief period of occlusion [3-6].

Fluctuating ptosis and ocular motility paresis in young patients are common presenting signs of ocular myasthenia gravis (OMG), but headache and macropsia are not expected. Though all tests for OMG are negative, we could not eliminate $\mathrm{OMG}$ in this patient since the diagnostic tests for OMG are often unable to display sensitivity and specificity simultaneously [12].

Vasculitis and demyelinating disease of central nervous system could also explain our patient's clinical signs. In these conditions however, findings usually have an acute and progressive course. These conditions were considered however. All infectious and immunologic markers were negative. The mild delay in the visual evoked potential latency and presence of a few, two millimeter, T2 hyperintense lesions in right frontotemporal region of cranial MRI might suggest multiple sclerosis but the number and location of the lesions do not explain patient findings.

Because of unilateral and bilateral abduction deficits, the neurologist suspected unilateral and bilateral sixth nerve paresis in this patient in two of the hospital admissions. In VI cranial nerve paresis or palsy, the esotropia is expected to increase in the field of lateral rectus action, and also on distance fixation. In our case the esotropia was increasing in the field of lateral rectus but contrary to expectations, it was decreased at distant fixation. These findings suggest that esotropia was not arising from the weakness of lateral rectus but from the overacting medial rectus.

Bilateral macropsia usually occurs in neurologic diseases, or as a side effect of drugs [13-14]. Unilateral macropsia or aniseikonia usually occurs in macular diseases or in anisometropic conditions [15-16]. This patient had neither of them. The pathogenetic mechanism for change in image size may have been a result of using varying degrees of accommodation in an effort to see clearly. 
Our patient's intermittent unilateral ptosis might be explained as an effort to close one eye to avoid diplopia. Similarly her occasional bilateral mild ptosis might be a result of trying see the objects more clearly using a stenopoeic slit or pinhole effect to reduce her accommodative effort.

The onset in this patient was unusual. Our patient was employed as an accountant, a career that demands close work with her eyes, and prolonged periods of accommodative strain. This strain on accommodation, without correction of her +4.00 diopter hyperopic refractive error, could explain her headaches and the variability in the motility pattern. The ataxia could have been caused by the diplopia.

The patient remained symptom-free immediately following the wearing of the prescribed lenses. During our follow-up period of 2.5 years, her symptoms did not return. Based on these observations, it is likely that the diagnosis is refractive accommodative esotropia, with a delayed onset. The delay in onset may have been precipitated by the occupational strains of her accounting job. Testing for OMG is not absolute, and the patient will be followed with this in mind.

The strength of this report is the thorough evaluation by a strabismologist who understands the characteristics of refractive accommodative esotropia. The weaknesses are the retrospective review of the clinical records which may contain erroneous patient history information or missing data.

In summary, signs and symptoms of refractive accommodative esotropia might simulate neurologic diseases in adults. Refractive accommodative esotropia should be considered when evaluating adults for diplopia associated with esotropia and headache. These patients benefit by combined evaluations of a neurologist and an ophthalmologist who is familiar with these conditions.

\section{References}

[1] Birch EE, Fawcett SL, Morale SE, Weakley DR, Wheaton DH. "Risk Factors for Accommodative Esotropia among Hypermetropic Children”. IOVS.2005;46(2), pp. 526-529.

[2] Von Noorden GK. "Accomodative Esotropia”. In: Craven L, Buckwalter W, Walter CP, editors. Binocular vision and ocular motility: Theory and management of strabismus, 5th ed. St Louis: Mosby, 1996, pp. 303-306.
[3] Swan KC. Esotropia following occlusion. Arch Ophthalmol. 1947;37:444-451.

[4] Von Noorden GK. "Acute acquired esotropia" In:Craven L, Buckwalter W,Walter CP, editors. Binocular vision and ocular motility Theory and management of strabismus,5th ed.St Louis: Mosby, 1996, pp. 324-325.

[5] Williams AS, Hoyt CS. "Acute comitant esotropia in children with brain tumors" Arch Ophthalmol. 1989; 107, pp. 376-378.

[6] Khan AO. Case Report: "Refractive accomodative esotropia following monocular patching" American Orthoptic Journal. 2004;54, pp.133-134.

[7] Burde RM, Savino PJ, Trobe JD. "Diplopia" In:Clinical decision in Neuro-opthalmology, 1st ed. St Louis: Mosby, 2002, pp. 158-197.

[8] Passo M, Shults WT, Talbot T, Palmer EA. "Acquired esotropia: a manifestation of Chiari malformation" J Clin NeuroophthalmoI . 1984; 4, pp. 151-154.

[9] Burian HM, Miller JE. Comitant convergent strabismus with acute onset. Am J Ophthalmol. 1958;45:55-64

[10] Simon JW, Waldman JB, Couture KC. Cerebellar astrocytoma manifesting as isolated, comitant esotropia in childhood. Am J Ophthalmol 1996;121:584-586.

[11] Bixenman WW, Laguna JF. Acquired esotropia as initial manifestation of Amold-Chiari malformation. J Pediatr Ophthalmol Strabismus 1987;24:83-86

[12] Mittal MK, Barohn RJ, Pasnoor M, McVey A, Herbelin L, Whittaker T, Dimachkie M. "Ocular myasthenia gravis in an academic neuro-opthalmology clinic: clinic features and therapeutic response" J Clin Neuromuscul Dis .2011;13(1), pp.46-52.

[13] Ghanizadeh, A. "Citalopram-induced macropsia" Clin Neuropharamcol. 2007; 30(4),pp. 246-247.

[14] Zwijnenburg PJ, Wennink JM,Laman DM, Linssen WH. "Alice in Wonderland syndrome: a clinical presentation of frontal lobe epilepsy” Neuropediatrics. 2002; 33, pp.53-5.

[15] Rutstein RP. "Retinally induced aniseikonia: a case serious" Optom Vis Sci.2012;89(11):50-55.

[16] Westheimer G. "First description of aniseikonia" $\mathrm{Br} \mathrm{J}$ Ophthalmol.2007;91: p.6. 